\title{
Analyses of the Secondary Cosmic Ray using CCD camera in high-altitude observatories and Antarctica stations
}

\author{
Guillaume HUBERT ${ }^{a, *}$ \\ ${ }^{a}$ ONERA DPHY, University of Toulouse \\ 2 av. Edouard Belin, Toulouse, France \\ E-mail: guillaume.hubert@onera.fr
}

\begin{abstract}
Charge-Coupled Device (CCD) and Array Pixel Sensors (APS) can be used to image radiationinduced energy deposition. The high sensitivity of depleted silicon to ionizing radiation constitutes an opportunity to investigate radiation effects while it is a nuisance to astronomer activities. CCD and APS provide a better combination of spatial and intensity resolution for radiation events than other available types of detector. This paper proposes to analyze radiation events observed in the CCD camera and more specifically analyses of charge deposition spectra and spatially extensive events. Measurements were performed in the Pic du Midi from 2011 to 2015 and in the Concordia Antarctica station since 2018. Coupled transport models (i.e. particle transport and charge transport in semiconductors) allow investigating contributions to charge collection spectra as a function of the particle nature, i.e. neutron, proton and muon. Coupled measurements and simulations allow to access to the detected secondary CR flux and the charge deposition pattern. Results showed that high charge level events seen on atmospheric sites can be considered as hadronic component (mainly neutrons and protons) while low charge levels and punctual events are induced by muons which are able to generate up to $3 \mathrm{fC}$ in the CCD camera. Hence, thanks to double level of measurement sites, muon discrimination from other secondary particles has been investigated. Cross-comparison analyses based on CCD and neutron spectrometers operated in both station/observatory investigate secondary CR dynamic.
\end{abstract}

37th International Cosmic Ray Conference (ICRC2021)

12-23 July 2021

Berlin, Germany - Online

"Speaker 


\section{Introduction}

When the primary cosmic rays penetrate the Earth's atmosphere, they interact with atmospheric nuclei and generate secondary radiation [1]. Products of showers are mainly neutrons, protons, heavy ions, pions and muons. Their intensities depend on the altitude, the geomagnetic latitude and the Sun's activity. In terms of atmospheric radiation effects, neutrons are considered as the most important contributions. Moreover, muons are the most numerous terrestrial species at sea level and they penetrate even meters of material.

The atmospheric radiation field characterizations are still a challenge; real-time radiation monitoring has become a general trend and several works have been performed [2]. Bonner spheres, scintillation counters and instruments based-on semiconductor detectors can be used to measure the atmospheric radiation field. Charge-Coupled Devices (CCD) and Array Pixel Sensors (APS) can be used to image radiation-induced energy deposition [3]. While it is a nuisance to astronomer activities, the high sensitivity of depleted silicon to ionizing radiation constitutes an opportunity to investigate radiation effects. Indeed, previous work has shown that CCD and APS devices can be used to investigate the small-scale interaction events of neutrons and protons [4]. Imagers provide a better combination of spatial and intensity resolution for radiation events than other available types of detector.

In this paper, measurements concern cosmic-ray induced neutrons using neutron spectrometer and charge transients in CCD. Multi-Physics modeling allows both measurement approaches to be correlated. Analyses of the charge levels in pixels induced by radiation events are proposed. Then, cross-comparisons allow investigating atmospheric radiation dynamic properties, including neutron/proton and muon contributions.

\section{Experimental and modelling platform}

\subsection{High-altitude observatories}

Scientific instruments such as a neutron spectrometer and CCD camera are operated in highaltitude observatories since 2011. The Pic-du-Midi observatory located in the French Pyrenees at $2885 \mathrm{~m}$ above sea level was the first since 2011, while the second was the Concordia station located since December 2015 at Dome C on the Antarctic Plateau ( $\left.75^{\circ} 06^{\prime} \mathrm{S}, 123^{\circ} 23^{\prime} \mathrm{E}\right)$ at height of 3,233 meters above the sea level. All details were presented in previous works [5][6].

\subsection{Imaging SEE monitor based on PIXIS2048 CCD}

A CCD is a large photoactive capacitor matrix that is capable of shifting the charge packet stored in each capacitor. A single particle can induce a signal in a cluster of adjacent pixels. This is because the charge induced by this particle is spreading out during the charge collection process and it can be finally collected by several adjacent pixels. The charge collected by each pixel can be measured and the total charge can be determined by summation of all fractional charges. In a CCD device, various noise sources must be considered, because some radiation events are characterized by low energies. It is important to know the noise contributions, in order to recover the useful signal. CCDs do have inherent noise sources: the dark current, the 
thermal noise and transfer noises. The main contribution to noise is due to the presence of a quiescent bias, input circuit noise introduced during sampling, thermally generated dark current and reset noise in the summing bus output lines. The thermal noise corresponds to the statistical dispersion of the dark current. In addition, charge transfer inefficiency (CTI), wherein a small fraction of the charge remains behind during cell-to-cell transfer, will limit performance. Thus, it is preferable to operate at very low temperatures, in order to minimize dark current and the thermal and transfer noises.

This work was performed in using the PIXIS2048 4.2 million-pixel $(2048 \times 2048)$ full frame CCD. Its pixel size is $13.5 \mu \mathrm{m} \times 13.5 \mu \mathrm{m}$ and the depth of the depletion region is $4 \mu \mathrm{m}$. Moreover, PIXIS2048 is a large format camera that offers cooling down to $-70^{\circ} \mathrm{C}$ with lifetime vacuum guarantee. The threshold will be different for each pixel; this is why we will have a 'threshold array' of the same size as the matrix of pixels. Since the threshold is different for each pixel, the system eliminates a large part of the non-uniformity of the pixel response, which is not homogeneous but reproducible. The charge level $\eta(x, y)$ induced by the radiation event in the cell $(\mathrm{x}, \mathrm{y})$ is given by Equation 1 .

$$
\eta(x, y)=\eta_{\text {pixel }}(x, y)-\eta_{\text {noises }}(x, y)
$$

where $\eta_{\text {pixel }}(x, y)$ and $\eta$ noises $(x, y)$ are the signal measured and the noise level deduced from the previous acquisition in the pixel $(\mathrm{x}, \mathrm{y})$. Then, the total charge level nevent induced by the radiation event is given by:

$$
\eta_{\text {event }}=\sum_{x, y}\left[\eta_{\text {pixel }}(x, y)-\eta_{\text {noises }}(x, y)\right]
$$

\subsection{Modelling platform}

The MUSCA SEP3 tool [7] is based on a multi-level physical approach to model all physical mechanisms from the incident particle to the charge-levels collected in the circuit level. The CCD camera and its environment (i.e. radiation and matter) were considered. The first step consists in modeling the atmospheric environment, i.e., the neutron, proton and muon radiation fields. Neutron spectra result directly from recorded neutron spectra, while muon and proton spectra were calculated using ATMOS CORE [8]. Transient effects are induced when a CR-induced particle (neutron, proton or muon) creates a secondary charged particle (or several particles), which crosses the active volume of a CCD. Primary or secondary ionization induces charge generation along the path of the particle and then an induced charge level in multiple pixels. The charge produced can be clocked out during readout and induce transient events. The transport/collection models are based on a diffusion model. The model used in MUSCA SEP3 considers two regions: the high-field region, characterized by an collection efficiency equal to $100 \%$, and the diffusion region (i.e. low-field region) characterized by an efficiency model deduced from simulations. The efficiency value depends on the distance between the charge generation and the high-field region. Fig. 2 presents the 
CDD modeling, including 3D pixel array geometries and collection models. The silicon substrate thickness is equal to $500 \mu \mathrm{m}$. Then MUSCA SEP3 allows the neutron, proton or muon interactions to be modeled, as well as transport/collection models, to determine the charge level in a pixel.

3D Pixel array

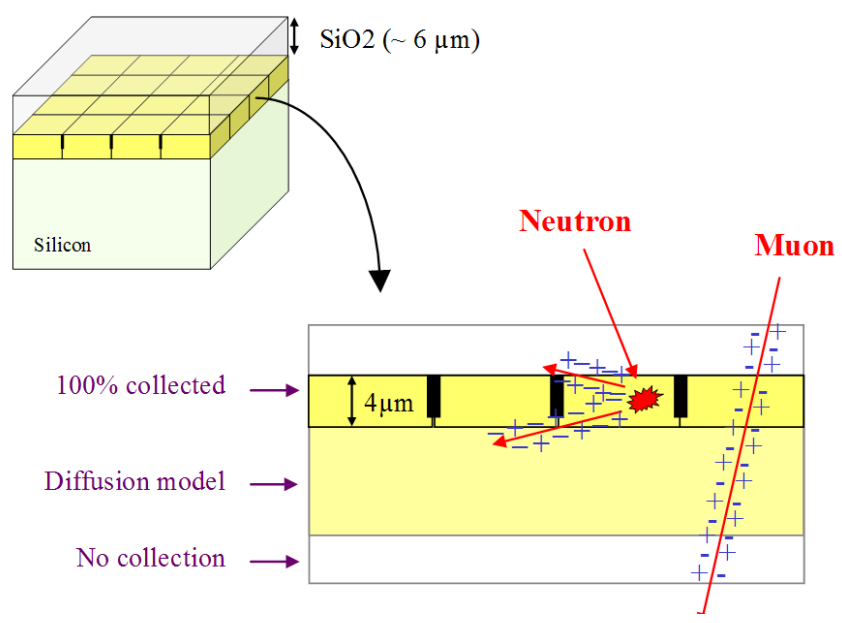

Fig. 1. CDD modeling including 3D pixel array and collection models.

\section{Results and analyses}

\subsection{Radiation event analyses}

Fig. 2 presents the cumumative CCD output event image obtained during the campaign operated in Concordia and five examples of spatially extensive events. The five events correspond to extended particle tracks, which probably relate to secondary ions emerging from a nuclear interaction. The analysis of the image shows that events are mainly charaterized by small area (one to few pixels) and low charge level. There are a small number of very intense events.

A significant event may represent a localized shower of secondaries from a high energy neutron or proton event just above the plane of the array. This event is characterized by two clusters, which correspond to a secondary ion including two light ions. $0.6 \mathrm{pC}$ was collected from these interactions. Secondary ions deposit approximately $0.15 \mathrm{pC}$ and $0.22 \mathrm{pC}$. This event corresponds to a nuclear reaction between a high energy proton or neutron and a silicon nucleus. Besides, both light ions are emitted horizontally in the pixel array. The pixel charge level treatment along the ion track allows the LET profile of the secondary ions to be deduced. It is not possible to deduce the incidental particle characteristics (i.e. nature, energy and direction) precisely. Nevertheless, analyses based on linear energy transfert characteristics and the nuclear neutron/proton databases suggest that a neutron or a proton with significant energy (several hundred $\mathrm{MeV}$ ) will be able to induce this secondary ion configurations. 
A similar spatially extensive event (Fig. 3) was observed in the Pic-du-Midi on Juanuary 2013. This event corresponds to a shower of secondaries from a high energy neutron or proton event just above the plane of the array. By analyzing nuclear properties of $n / p-$ Silicon nuclear interaction, it is possible to assess the order of magnitude of the probability that secondary ions characterized by two protons whose energies are of about $5-10 \mathrm{MeV}$ and with direction properties similar to those of the investigated event (i.e., in the plane of the array). Thus, the probability of inducing an event similar to the event presented in Fig. 4 is estimated to be $2.8 \times 10^{-8}$ events/s. As a matter of fact, one event was observed over a period of two years of measurement, i.e. $\sim 2 \times 10^{-8}$ events $/ \mathrm{s}$. As a consequence, the calculated probability is consistent with the operational occurrence and confirms the nature and characteristics of the event.
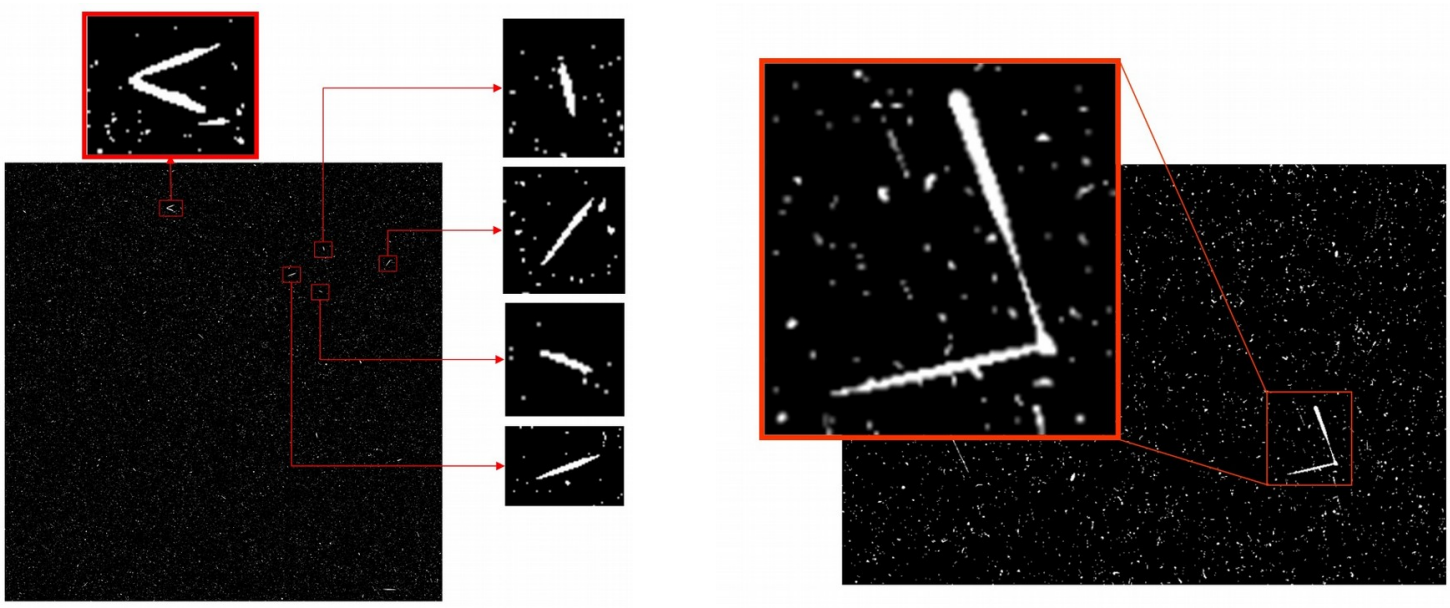

Fig. 2. Radiation particle interaction eventsFig. 3. Radiation particle interaction events observed in separate image frames in Concordiobserved in separate image frames in te Pic-du-Midi, during the 2017-18 wintering operations. occurring in January and February 2013.

\subsubsection{Charge-event spcetrum analyses}

Fig. 4 presents the charge-event spectra obtained from measurements performed in the Concordia station, (b) in the Pic-du-Midi observatory and (c) in the laboratory (ground) located in toulouse city. The time integration is in the order of 2 weeks for the three spectra. The spectra obtained in Concordia and at the Pic du Midi have a fairly similar form, while the spectrum obtained in Toulouse with a lower component for the high charge event levels. This is due, firstly because the event statistic is lower to the ground (considering an equivalent integration time), and the other laboratory is in the basement with a 4-stage screening. The neutrons and protons fields, probably responsible for the high charge event, are attenuated while muon field inducing low charge event is weakly impacted. 

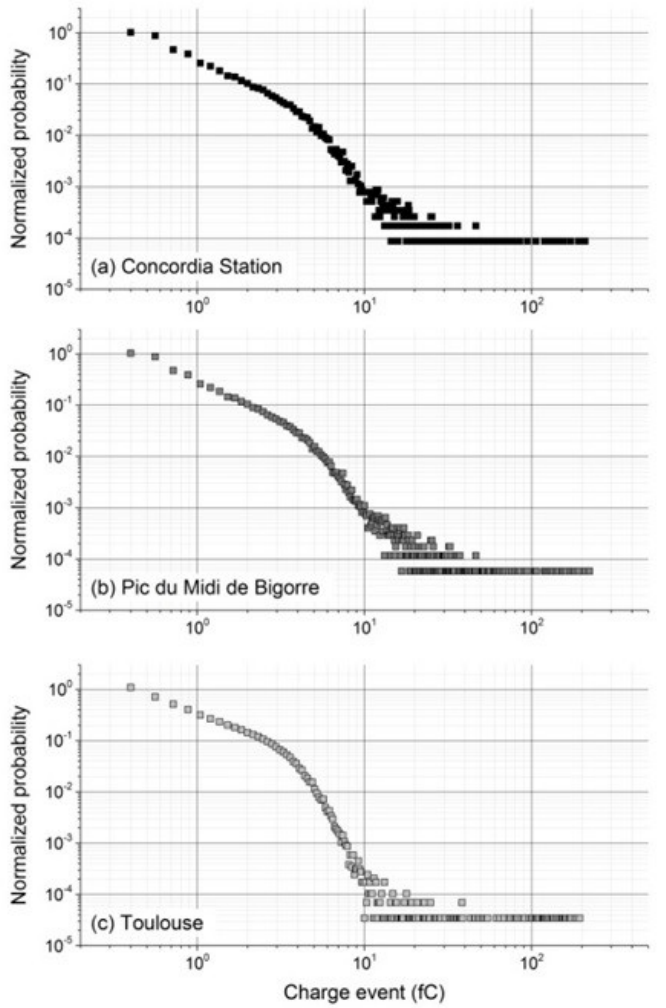

Fig. 5: charge-event spectra obtained from measurements performed in the Concordia station, (b) in the Pic-du-Midi observatory and (c) in the laboratory (ground) located in toulouse city.

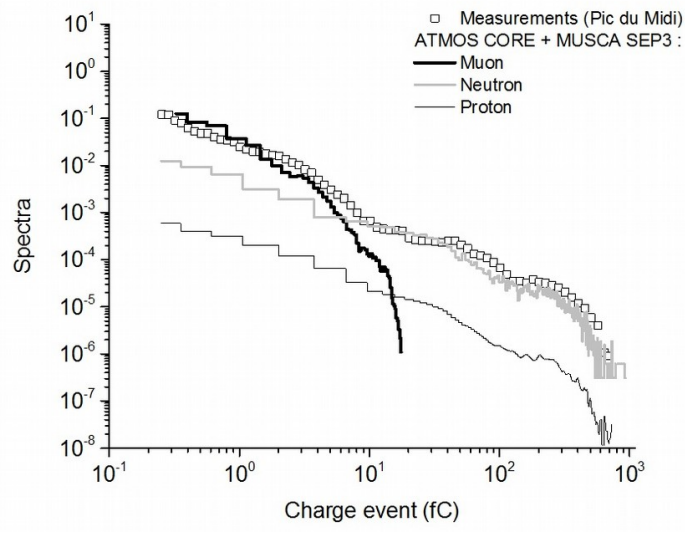

Fig. 6: Charge-collection spectrum obtained from measurements performed in the Pic du Midi over a long period of three years and compared to ATMOS CORE + MUSCA SEP3 simulations for neutron, proton and muon contributions.

Simulations can be performed by ATMOS CORE/MUSCA SEP3 to deduce the chargecollection spectra of neutron, proton and muon contributions. Thus, Fig. 5 presents the the charge-collection spectrum obtained from measurements performed in the Pic du Mid over a period of three years, and results issed from simulations. A good agreement has been obtained between measurements and modeling for the total contribution. The neutron environment used in our calculations is obtained using neutron spectrometer measurements, while proton and muon environments are deduced from ATMOS CORE calculations. Modeling allows the contributions to the charge collection spectrum as a function of the particle nature (neutron, proton or muon) to be investigated. Thus, the high charge event levels (typically more than 10fC) are induced by neutrons and protons, while low charge event levels are induced by muons (some fC). These results suggest an interesting issue, which consists in discriminating the neutrons/protons and muons: charge events lower than $8 \mathrm{fC}$ can be considered as induced by muons and charge events greater than $8 \mathrm{fC}$ are the consequence of protons or neutrons. This result allows a discrimination approach to investigate the neutron/proton and muon fluxes to be proposed. 


\subsection{Atmospheric radiation dynamic properties}

Results previously presented suggest that muon and proton/neutron contributions can be extracted from the CCD camera measurements. Fig. 7 presents the relative neutron and muon flux variations issued from the CCD measurements (i.e. event charge $<8 \mathrm{fC}$ for the muon) and in using the neutron spectrometer. Analyses were performed over a oneyear period. However, the integration time is chosen as 3 days and the variation value is considered with respect to the average value during the measurement period. The cross comparison shows a good correlation between the neutron flux obtained from the CCD and that derived from the neutron spectrometer. This demonstrates the capability of investigating the neutron flux dynamics with two complementary detector types. It is interesting to note that the neutron variations are significant (i.e., decrease) during the winter season. This is due to the snow, which mainly affects the neutron flux. The neutron variations are significant during winter, while the muon variations are similar throughout the entire year. Indeed, muons are not affected by the snow and hydrometric conditions.

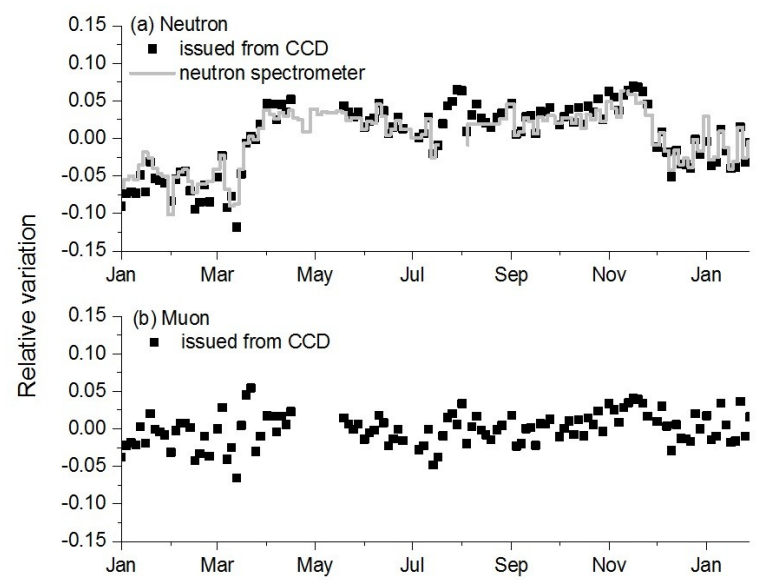

Fig. 7: (a) Relative neutron flux (En $>15.9 \mathrm{MeV}$ ) variations obtained from the neutron spectrometer and neutron flux variations obtained from the CCD data over the period of one year. (b) Relative muon flux variations obtained from the CCD camera over the period of one year.

\section{Conclusion}

This paper describes analyses based on two radiation detectors, i.e., a CCD camera and a neutron spectrometer, and a modeling approach that allows measurements to be relied on.

These investigations require the use of a modeling approach, i.e., to consider the radiation environment (neutron sepctrometer and/or ATMOS CORE), interaction processes in matter and SEE mechanisms (mainly transport and collection). Methodology based-on MUSCA SEP3 allows relevant cross-comparisons to be performed. MUSCA SEP3 modeling was performed to investigate contributions to 
charge collection spectra as a function of the particle nature, i.e., neutron, proton or muon. Results show that high charge level events (typically more than $10 \mathrm{fC}$ ) are induced by neutrons and protons, while low charge level events are more probably induced by muons (i.e., some fC). Hence, discrimination between the neutron/proton and muon components from CCD camera measurements has now been established.

Analyses show that identical neutron variations can be deduced from the neutron spectrometer and the CCD camera. Secondly, they illustrate the correlation between neutron/proton and muon environments using CCD camera measurement analyses. Finally, this paper demonstrates the interest in coupling measurements and modeling, i.e., it allows the radiation event characteristics to be investigated on the one hand and the dynamic properties in complex radiation fields, such as the atmospheric natural radiative environment, on the other hand. Long-term measurments will be accumulated in the Concordia station in the framework of the CHINSTRAP project.

\section{Acknowledgments}

The authors thank the personnel of the Concordia station for their great help and hard work in harsh conditions. This work is supported by the IPEV (French Polar Institute, Institut Paul-Emile Victor) in the framework of the CHINSTRAP project.

\section{References}

[1 J. F. Ziegler, “Terrestrial Cosmic Rays,” IBM J. Res. Develop., Vol. 40, No. 1, January 1996.

[2] P. Goldhagen, et al., "Recent results from measurements of the energy spectrum of cosmic-ray induced neutrons aboard an ER-2 high-altitude airplane and on the ground," Advanced Space Research, Vol. 32, No 1, pp. 35-40, 2003.

[3] W. Shedd and B. Buchanan, “Transient radiation effects in CCD's," IEEE Trans. Nucl. Sci., Vol. 23, No. 6, Dec. 1976.

[4] Z. Török and S. P. Platt, "Application of imaging systems to characterization of single-event effects in high-energy neutron environments,” IEEE Trans. Nucl. Sci., 53(6), pp. 3718-3725, Dec. 2006.

[5] G. Hubert et al., Modelling of ground albedo neutrons to investigate seasonal cosmic-ray-induced neutron variations measured at high-altitude stations. J. Geophys. Res. Space Physics, 121, 186201,2016

[6] G. Hubert, Analyses of cosmic ray induced-neutron based on spectrometers operated simultaneously at mid-latitude and Antarctica high-altitude stations during quiet solar activity. Astroparticle Physics Journal, 83, 30-39, 2016.

[7] G. Hubert et al., "Operational SER calculations on the SAC-C orbit using the Multi SCAles Single Event Phenomena Predictive Platform (MUSCA SEP3)”, IEEE Trans. Nucl. Sci., Vol. 56, No.6, pp. 3032-3042, Dec. 2009.

[8] G. Hubert, S. Aubry, "Simulation of atmospheric cosmic-rays and their impacts based on precalculated databases, physical models and computational method", Journal of Computational Science, 2021. 